\title{
Polycyclic Aromatic Hydrocarbons in Agra, India: Gas-particle partition, concentration and potential risk
}

\author{
PunEet Kumar VERMA ${ }^{1}$, Dinesh SAH ${ }^{1}$, K. MAHARAJ \\ KUMARI $^{1}$ AND ANITA LAKHANI ${ }^{1}$ \\ ${ }^{1}$ Dayalbagh Educational Institute, Agra 282005, India \\ anitalakhani01@gmail.com
}

This study investigated the gas-particulate partitioning of Polycyclic Aromatic Hydrocarbons and Nitro-PAHs using gas and particulate phase samples collected at a traffic dominated site in Agra India, from October 2015 to July 2016. The concentration of PAHs in the gas and particulate phase were $1732.5 \pm 175.1$ and $3121 \pm 308.9 \mathrm{ng} \mathrm{m}^{-3}$ respectively; whereas the gas and particle phase concentration of Nitro-PAHs were $24.6 \pm 6.55$ and $65.3 \pm 16.3 \mathrm{ng} \mathrm{m}^{-3}$ respectively. The experimental values of Pankow model shows statistically significant correlation between experimentally determined $\log K p$ and modelled log $K p$. Log $K p$ of each PAH was correlated with sub-cooled liquidvapour pressure $\left(p^{\circ} L\right)$ of each PAH resulting in slope of -0.60 and $\mathrm{r}^{2}=0.82$. Regression statistics for $\log K p$ vs $\log p^{\circ} l$ indicates towards primary emissions and shows that PAHs does not get enough time to get partitioning in both the phase after emission. Effect on partitioning due to absorption of PAHs in organic layer on the particulate surface was estimated using Octanol-air partitioning model. Statistically significant correlation for $\log K p$ and $\log K_{O A}$ was obtained, with an average value of correlation coefficient of 0.62 , slope of 1.71 and intercept -1.61 . Octanol-air model results indicate that, $K_{O A}$ can be used as a predicting parameter for the gasparticle partitioning process of PAHs in the atmosphere. Both the adsorption and absorption model indicate towards local emissions. Lifetime risk of cancer (ILCR) model was used to assess the health risk of $\sum$ PAHs and the results indicates that the adults and children in Agra are more prone to cancer risk in comparison to infants via dermal exposure. 